\title{
Association between CYP19A1 polymorphisms and sex hormones in postmenopausal Japanese women
}

\author{
Kumiko Kidokoro ${ }^{1,2}$, Kazuhiko Ino ${ }^{2}$, Kaoru Hirose ${ }^{3}$, Hiroaki Kajiyama ${ }^{2}$, Satoyo Hosono ${ }^{1,2}$, Takeshi Suzuki ${ }^{3}$, \\ Takakazu Kawase $^{3}$, Akio Hiraki ${ }^{3}$, Nobuyuki Hamajima ${ }^{4}$, Hideo Tanaka ${ }^{1,5}$, Kazuo Tajima ${ }^{1}$, Fumitaka Kikkawa ${ }^{2}$ \\ and Keitaro Matsuo ${ }^{1,5}$
}

In postmenopausal women, extraovarian sex hormone production plays an important role in hormone-related diseases, such as breast and endometrial cancers. Aromatase, an enzyme encoded by CYP19A1, is a key enzyme involved in estrogen biosynthesis. The impact of CYP19A1 polymorphisms on serum sex hormone levels in the Japanese population has never been investigated. This study enrolled 100 postmenopausal Japanese women found to be without cancer. Twenty-five CYP19A1 loci were identified, and measurements were conducted on serum levels of sex hormones; lifestyle data were collected, namely estrone (E1), estradiol (E2), testosterone and sex hormone-binding globulin (SHBG). We conducted a cross-sectional analysis to evaluate the impact of CYP19A1 haplotype on serum sex hormone levels. We found that subjects with $\mathrm{BMI} \geqslant 25 \mathrm{~kg} / \mathrm{m}^{2}$ showed a significant difference in circulating testosterone levels $(0.29 \pm 0.19, P=0.050)$. Neither age nor the amount of physical exercise or drinking habits showed any effect on hormone levels. We identified seven haplotype blocks in CYP19A1 by LD analysis. Estrone levels differed in rs12148604 (SNP 1) and rs11632903 (SNP14). No significant locus for estradiol was observed. SHBG levels were associated with rs4441215 (SNP11). Testosterone levels were strongly associated with rs752760 (SNP24) and rs2445768 (SNP25) and weakly associated with SNP 1, SNP11 and SNP14 as well. We found that polymorphisms in CYP19A1 influence sex hormone levels in Japanese postmenopausal women.

Journal of Human Genetics (2009) 54, 78-85; doi:10.1038/jhg.2008.11; published online 16 January 2009

Keywords: CYP19A1; polymorphism; aromatase; sex hormone; lifestyle; endometrial cancer

\section{INTRODUCTION}

An association has been recognized between sex hormones and the risk of breast, ovarian and endometrial cancers, particularly in postmenopausal women. Earlier studies have shown a clear association between the circulating levels of estrogen and testosterone in postmenopausal women and the risk of these cancers. ${ }^{1-3}$ These findings highlight the importance of identifying factors that define hormonal levels in postmenopausal women.

On reaching menopause, the ovaries cease to produce endogenous estrogens, at which point extragonadal sites such as adipose tissues become a major alternative source. ${ }^{4-6}$ Estrogen production occurs through the catalytic activity of the aromatase enzyme, which is encoded by CYP19A1. On the assumption that certain CYP19A1 polymorphisms might affect estrogen levels, several studies have explored the potential impact of polymorphisms in CYP19A1 on the risk of several types of cancer in Caucasian populations. ${ }^{7-9}$ More recently, Haiman et al. ${ }^{10}$ examined 103 single nucleotide polymorphisms (SNPs) in more than 3000 cancer-free subjects of mainly
European descent $(93 \%)$ and found an association between haplotypetagging SNPs in the $5^{\prime}$ region and circulating levels of estrogen in CYP19A1. Dunning et al. ${ }^{11}$ examined two loci in CYP19A1 and found a significant association between these two loci and sex hormone levels. Although hormone-related cancer incidence is gradually increasing in Asian populations, including the Japanese ${ }^{12}$ information regarding the effect of CYP19A1 SNPs on estrogen levels is still strongly lacking.

In this study, we examined the association between the CYP19A1 polymorphisms in conjunction with environmental elements and serum hormone levels in postmenopausal Japanese women.

\section{MATERIALS AND METHODS}

Subjects

This study enrolled 100 cancer-free naturally postmenopausal women through the Hospital-based Epidemiologic Research Program at Aichi Cancer Center (HERPACC) between December 2005 and June 2006 as a part of the Japan Multi-institutional Collaborative Cohort Study (J-MICC). ${ }^{13}$ Subjects visited ACC for more intensive examination after testing positive in a cancer screening

${ }^{1}$ Division of Epidemiology and Prevention, Aichi Cancer Center Research Institute, Nagoya, Japan; ${ }^{2}$ Department of Gynecology and Obstetrics, Nagoya University Graduate School of Medicine, Nagoya, Japan; ${ }^{3}$ Department of Planning and Information, Aichi Prefectural Institute of Public Health, Nagoya, Japan; ${ }^{4}$ Department of Preventive Medicine/ Biostatistics and Medical Decision-Making, Nagoya University Graduate School of Medicine, Nagoya, Japan and ${ }^{5}$ Department of Epidemiology, Nagoya University Graduate School of Medicine, Nagoya, Japan

Correspondence: Dr K Matsuo, Division of Epidemiology and Prevention, Aichi Cancer Center Research Institute, 1-1 Kanokoden, Chikusa-ku, Nagoya 464-8681, Japan. E-mail:kmatsuo@aichi-cc.jp

Received 3 October 2008; revised 6 November 2008; accepted 7 December 2008; published online 16 January 2009 
test, but were eventually found to not have cancer. All subjects consented to blood sampling for the measurement of hormone levels and genetic assay. Subjects currently receiving any kind of hormonal therapy were excluded. In the HERPACC, all first-visit outpatients at ACC Hospital (ACCH) are asked to fill out a self-administered questionnaire and provide a 14-ml blood sample after the written informed consent is obtained. All questionnaires are then checked by trained interviewers for incomplete responses. Details of the HERPACC study are described elsewhere. ${ }^{14}$ This study was approved by the ethics committee at ACC.

\section{Measurement of hormone levels}

Hormone level analyses were carried out on serum samples stored at $-80^{\circ} \mathrm{C}$. Levels of estradiol, estrone, testosterone and sex hormone-binding globulin (SHBG) were measured by SRL Inc. (Tokyo, Japan). Estrone levels were measured by radioimmunoassay (Teikokuzoukiseiyaku Medical, Tokyo, Japan), estradiol and testosterone levels by electrochemiluminescent immunoassay (Roche Diagnostics, Tokyo, Japan) and SHBG levels by immunoradiometric assay (Siemens Medical Solutions Diagnostics, Tokyo, Japan).

\section{SNP selection, genotyping and analysis for linkage disequilibrium (LD)}

The SNP loci examined were basically selected by SNP Browser ver. 3.5 (Applied Biosystems, Foster City, CA, USA) ${ }^{15}$ on the basis of the HapMap database for the Japanese in Tokyo (build 36) ${ }^{16,17}$ Selection criteria for each locus were (1) minor allele frequency (MAF) greater than $30 \%$ and (2) haplotype $R^{2}$-value greater than 0.95 . Of 169 candidate SNPs, 82 were excluded on the basis of MAF criteria. Of the remaining 87 SNPs, the 25 SNPs that were able to tag the other 62 SNPs owing to strong LD were finally selected.

DNA was extracted from the buffy coat fraction in each subject using a Blood Mini Kit (Qiagen KK, Tokyo, Japan) and analyzed using the polymerase chain reaction (PCR) TaqMan method ${ }^{18}$ with the 7500 fast real-time PCR System (Applied Biosystems). The probes used were specifically designed for rs12148604, rs2899473, rs12900487, rs1865803, rs10459592, rs12591359, rs767199, rs16964221, rs12908960, rs7172156, rs4545755, rs11636403, rs3889391, rs2414101, rs17647707, rs17647719, rs1902586, rs936306, rs999480, rs2470152, rs3751591, rs1004982, rs1902585, rs752760 and rs2445768. Genotyping was conducted in duplicate in cases where accordance with the Hardy-Weinberg equilibrium (HWE) was violated.

LD was evaluated by means of $\mathrm{LD}$ coefficients $\left(D^{\prime}\right)$. Haplotype blocks were defined using $95 \%$ confidence intervals of $D^{\prime}$, where the upper and lower boundaries for strong LD were set as 0.98 and 0.60 , as proposed by Gabriel et al..$^{19}$ Minimum $R^{2}$-value for strong $\mathrm{LD}$ was set as 0.30 . Haplotype alleles and their frequencies were estimated using SNPAlyze (Dynacom, Yokohama, Japan) on the basis of multiple SNPs obtained with the expectation-maximization algorithm.

\section{Exposure data}

Consumption of types of alcoholic beverages (Japanese sake, beer, shochu, whiskey and wine) was determined with reference to the average number of drinks per day, which was then converted into a Japanese sake (rice wine) equivalent. ${ }^{20}$ The drinking habit was further classified into the four categories of never, former, moderate and heavy drinking. Heavy drinkers were those currently drinking alcoholic beverages 5 or more days per week at $46 \mathrm{~g}$ (two 'go') or more per day, whereas moderate drinkers were those currently consuming less frequently 5 days per week. Former drinkers were defined as those who had quit drinking at least 1 year before the survey. Body mass index (BMI) was calculated as self-reported weight $(\mathrm{kg})$ divided by the square of self-reported height $(\mathrm{m})$. A validation study showed the correlation coefficient for BMI to be $0.92 .^{21}$

The questionnaire also enquired about regular physical exercise. Subjects were asked to report the frequency and intensity of recreational exercise. In this study, subjects who reported a frequency of at least once a month were defined as those who exercised.

\section{Statistical analysis}

Intergroup comparison was conducted using one-way ANOVA followed by pairwise comparison across groups. All statistical analyses were performed using Stata Ver. 10 (Stata Corp., College Station, TX, USA). A $P$-value of $<0.05$ was considered as statistically significant, and a value between 0.05 and 0.1 was considered as marginally significant.

\section{RESULTS}

Table 1 shows hormone and SHBG levels at baseline. We found significantly higher levels of circulating testosterone $(P=0.050)$ in subjects with $\mathrm{BMI} \geqslant 25 \mathrm{~kg} / \mathrm{m}^{2}$ than in subjects with $\mathrm{BMI}<25$. In contrast, no differences were observed for estrone, estradiol or SHBG levels. We found no significant differences across groups in levels examined according to age, amount of physical exercise or drinking.

Figure 1 shows the location of CYP19A1 on chromosome 15 with the distribution of 25 SNPs examined (a), and the genetic structure of CYP19A1 with untranslated first exons (b). SNP23 and SNP17 violated HWE, and interpretation for these loci should therefore be approached with caution. Table 2 shows hormone and SHBG levels according to the 25 SNPs examined and Table 3 displays a detailed analysis for loci showing significant association in Table 2. Estrone levels were significantly different in SNP1 (rs12148604) located in the 3'-flanking region of CYP19A1. Estrone levels rose with an increased number of $\mathrm{T}$ alleles at this locus in any of the models. Further, the $\mathrm{C}$ allele of SNP14 (rs2414101) located in intron 1 showed a significant difference in estrone levels in recessive and codominant models. No SNP showed a significant association with a change in estradiol levels. SHBG levels showed a significant difference with the G allele of SNP 11 (rs11636403) in intron 1 in all three models. Testosterone levels were significantly different with the A allele of SNP24 (rs752760) in exon 1 and T allele of SNP25 (rs2445768) in the $5^{\prime}$ near-gene region. SNP1, SNP11 and SNP14 also showed weak but significant association with testosterone levels.

The results of the LD analysis are shown in Figure 2. We identified seven haplotype blocks. Table 4 lists haplotypes on the basis of selected SNPs in each block along with their frequencies, as well as associations between hormone and SHBG levels and haplotypes in each block. Results showed a statistically significant difference in testosterone levels in block $7(P=0.0066)$. The haplotype $\mathrm{G}-\mathrm{T}-\mathrm{C}$ showed relatively smaller values compared with others. Blocks 1 and 5 showed marginally significant associations with regard to estrone levels $(P=0.0795$ and 0.0995 , respectively). In contrast, this haplotype block-based analysis revealed no remarkable findings with regard to estradiol and SHBG levels.

\section{DISCUSSION}

In this study, we identified seven haplotype blocks in CYP19A1 by assessing 25 tag SNPs. We further examined the possible association between haplotypes/genotypes and the levels of sex hormones and SHBG in postmenopausal Japanese women. Results showed that estrone levels were significantly affected by SNP1 (rs12148604) in the $3^{\prime}$ region and by SNP14 (rs11632903) in the region between untranslated exons I.2 and I.6. These loci were weakly but significantly associated with testosterone levels. Moreover, testosterone levels were markedly different across haplotypes in block 7 as well as with SNP24 and SNP25 (rs752760, rs2445768) located in the $5^{\prime}$ region of CYP19A1. Estrone and testosterone levels were higher in subjects with higher BMI, particularly with regard to testosterone.

We found a significant association between estrone levels and SNP1 genotype located in the $3^{\prime} \mathrm{UTR}$. This finding is consistent with earlier studies that showed an association between polymorphisms in the $3^{\prime}$ UTR (rs10046) and estrone and estradiol levels. ${ }^{10,11,22}$ According to the HapMap database, SNP1 (rs12148604) and rs10046 are in LD in 
Table 1 Characteristics and serum concentrations of hormones and SHBG

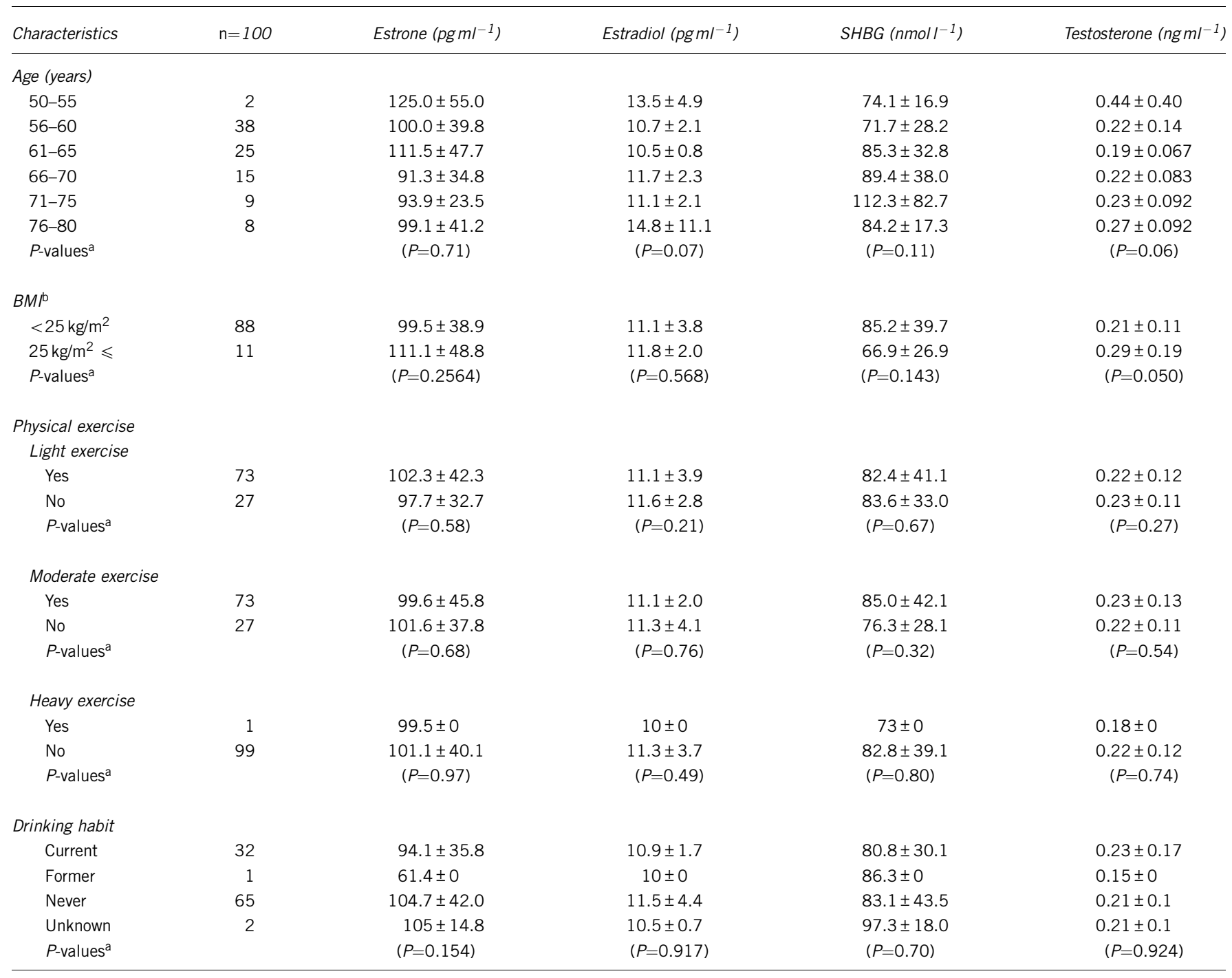

a $P$-values were test for equality by ANOVA test.

bone subject was excluded because of the lack of BMI information.

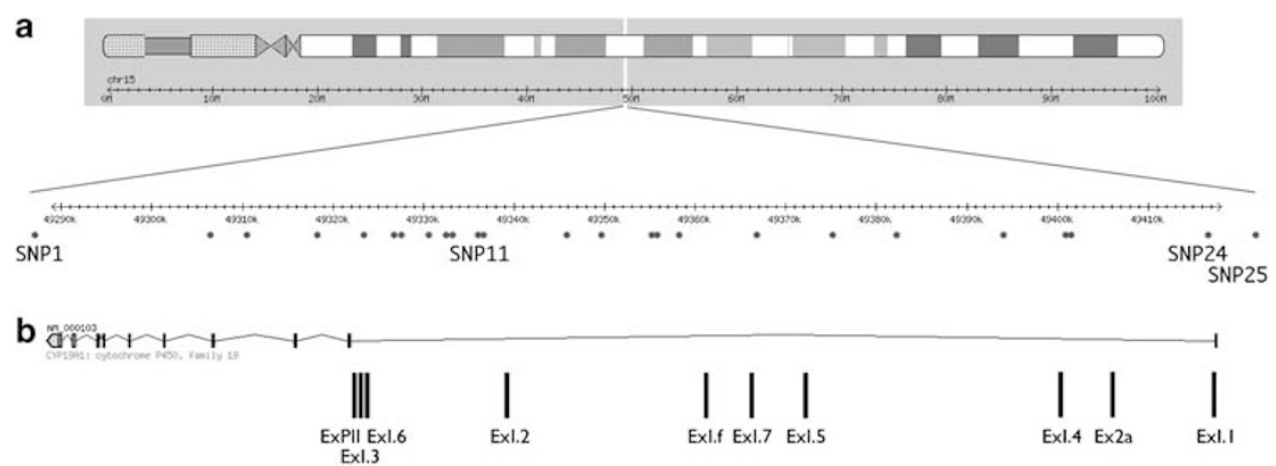

Figure 1 Overview of CYP19A1. (a) Ideogram of CYP19A1 and distribution of 25 SNPs. (b) Genetic structure of CYP19A1. Bars along the bottom represent untranslated first exons.

four ethnicities: JPT (Japanese individuals from Tokyo), HCB (Han Chinese individuals from Beijing), CEU (American individuals from Utah with northern and western European ancestry) and YRI (Yoruba individuals from Ibadan and Nigeria). Therefore, our results for SNP1, in combination with those of other studies, suggest the presence of a functional region in the $3^{\prime} \mathrm{UTR}$ of CYP19A1, which influences hormone levels among postmenopausal women of various ethnic backgrounds. 
Table 2 Concentrations of hormones and SHBG according to tagSNPs

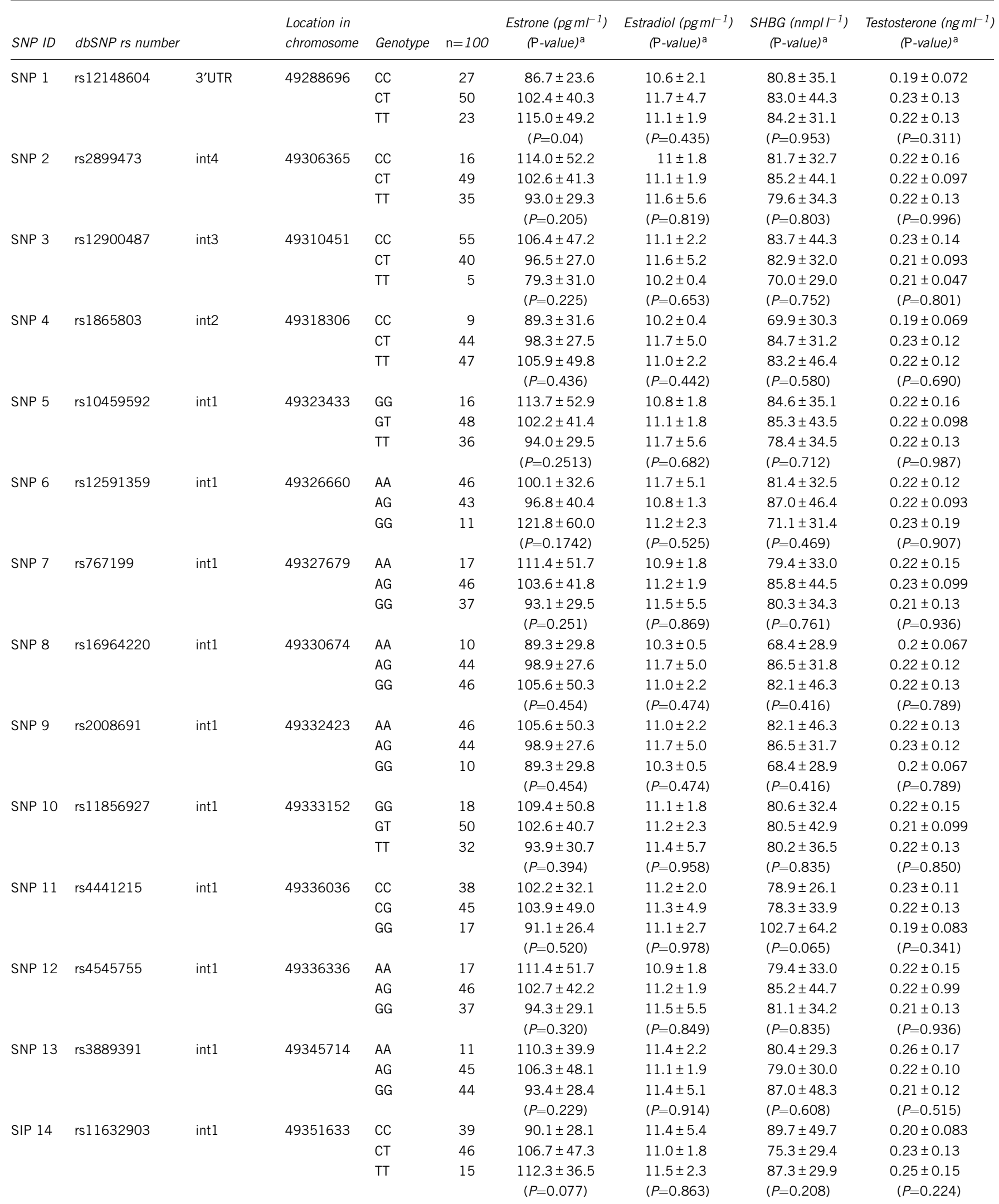


Table 2 Continued

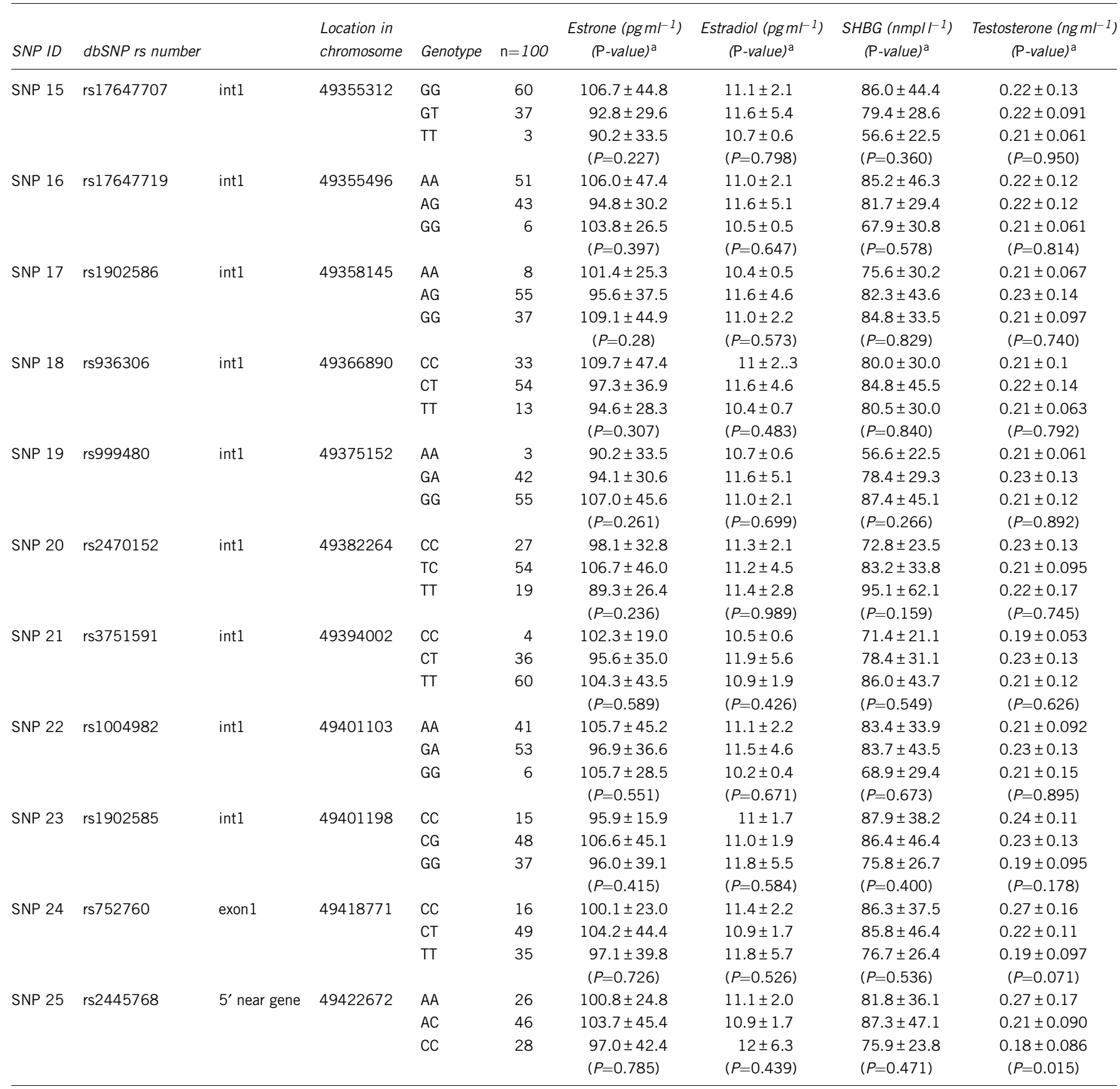

aANOVA test was used.

Regarding estrone levels, Cai et al. ${ }^{23}$ recently reported that the association between CYP19A1 SNPs (rs28566535, rs730154 and rs936306) were associated with estrone levels in the Chinese population. They found that the region of these loci is close to untranslated exon I.4 and includes a specificity protein-1-binding site, $(\gamma)$-interferon-activating sequence and glucocorticoid response element, which are essential for aromatase expression from I.4. However, we did not find any association with any of the loci we examined. Moreover, we found SNP14 (rs11632903) for estrone levels. The inconsistency may be explained partly by the difference of baseline estrone levels between our population (mean: $101.1 \mathrm{pg} \mathrm{ml}^{-1}$ ) and the Chinese population $\left(16.8 \mathrm{pg} \mathrm{ml}^{-1}\right)$. This might reflect genetic differences in other enzymes involved in estrogen synthesis between the two populations. Meanwhile, replication studies in other Asian populations are required.

We also found an association between testosterone levels and SNP24 (rs752760) and SNP25 (rs2445768) located in the $5^{\prime}$ noncoding region of CYP19A1. Several studies, conducted primarily in 
Table 3 Detailed analyses of selected loci for estrone, SHBG and teststerone levels

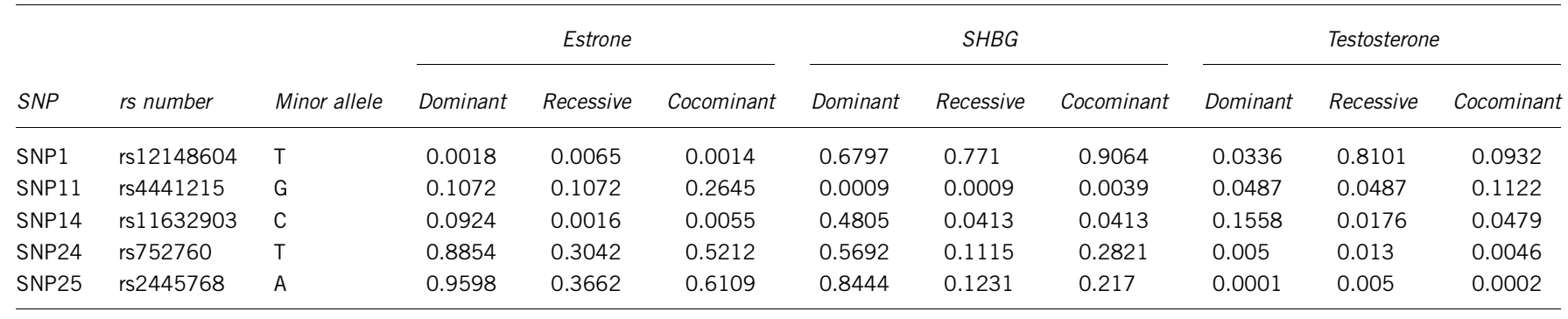

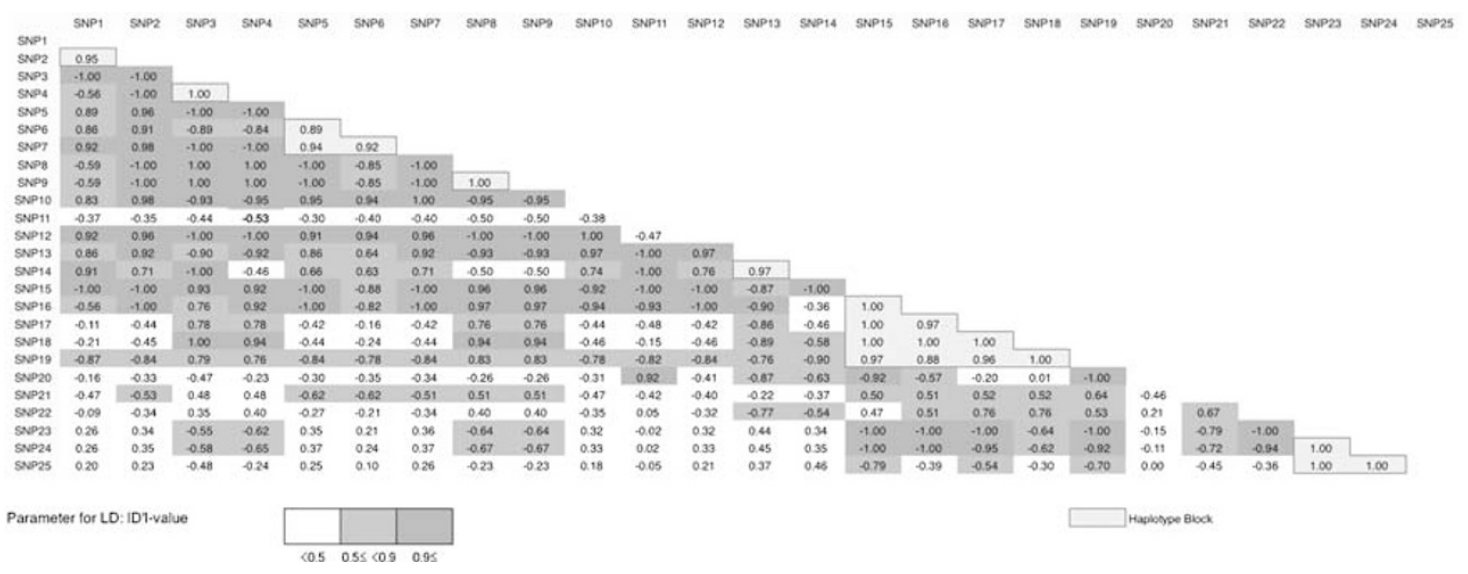

Figure 2 LD plot across the CYP19A1 loci. Cells feature pairwise $D^{\prime}$ values. Rectangles filled with red, pink and white correspond to LD magnitude of $\left|D^{\prime}\right|>0.9,0.9>\left|D^{\prime}\right| \geqslant 0.5$ and $\left|D^{\prime}\right|<0.5$, respectively. Groups of rectangles outlined in red represent haplotype blocks as estimated by SNPAlyze (Dynacom, Yokohama, Japan). The color reproduction of this figure is available on the html full text version of the manuscript.

Caucasian subjects, have examined the association between testosterone levels and CYP19A1 polymorphisms, although to our knowledge, none have found any association with $5^{\prime} \mathrm{UTR}$ loci. ${ }^{10,24,25}$ SNP24 is known to locate in the region containing the placenta-specific promoter (I.1 and I.2a). Considering that the subjects in this study were postmenopausal women, there is thus far no biological evidence supporting the possible contribution of promoters to testosterone levels. These findings, however, suggest the presence of functional SNPs around SNP24 and SNP25, which lead to differences in testosterone levels specific to the Japanese population. A weak but significant association between testosterone levels and SNP1, SNP11 and SNP14 is also suggestive of other functional regions. These findings warrant further studies into the underlying cause of these differences.

Although recent advances in genotyping have enabled an examination of the association between specific phenotypes, the study of hormone levels in postmenopausal women and potential loci by whole-genome SNP typing, as carried out in this study, is costly and susceptible to low statistical power. It is, therefore, more feasible to conduct association studies examining targeted genes on the basis of known biological mechanisms with tag SNPs. Haiman et al. ${ }^{10}$ recently conducted a tag SNP-based large scale cross-sectional study in postmenopausal Caucasian women and found a significant association between specific haplotype blocks and hormone levels. Our study is the first to apply a tag SNP-based approach to examine the impact of CYP19A1 SNPs on hormone levels in postmenopausal Japanese women. Although the function of loci showing a significant association requires further clarification, our study showed the effectiveness of this tag SNP-based approach in examining hormone levels in postmenopausal women.

In premenopausal women, the main source of estrogen is estradiol produced in ovarian granulose cells during the follicular phase, although fibroblasts in adipose tissue expressing aromatase are an alternate source. After menopause, however, adipose tissue becomes the major source of estrogen, and estrogenically weak estrone is produced from adrenal androstenedione. ${ }^{26,27}$ Moreover, at least half of this peripherally produced estrone is eventually converted to estradiol in extraovarian tissue. ${ }^{28}$ Testosterone is the precursor of estradiol produced in the ovary by the cholesterol metabolism pathway. It accumulates after menopause with the loss of the ovarian function. Our observation that the estrone and testosterone levels are higher in women with higher BMI than in those with lower BMI is consistent with the current knowledge of the metabolism of hormones in postmenopausal women. Studies conducted in other populations have also shown consistently increased hormone levels in accordance with higher BMI. ${ }^{26,29-31}$ Epidemiologically, obesity is a risk factor of hormone-dependent carcinoma, given the findings that the incidence of breast, endometrial and ovarian cancer is increased in postmenopausal women, along with increased levels of estrogen production in adipose tissues. ${ }^{32-35}$ On this basis, identifying those CYP19A1 loci that influence hormone levels may be an effective indicator of patients susceptible to hormone-related cancers. 
Table 4 Haplotype frequencies according to haplotypes in seven blocks

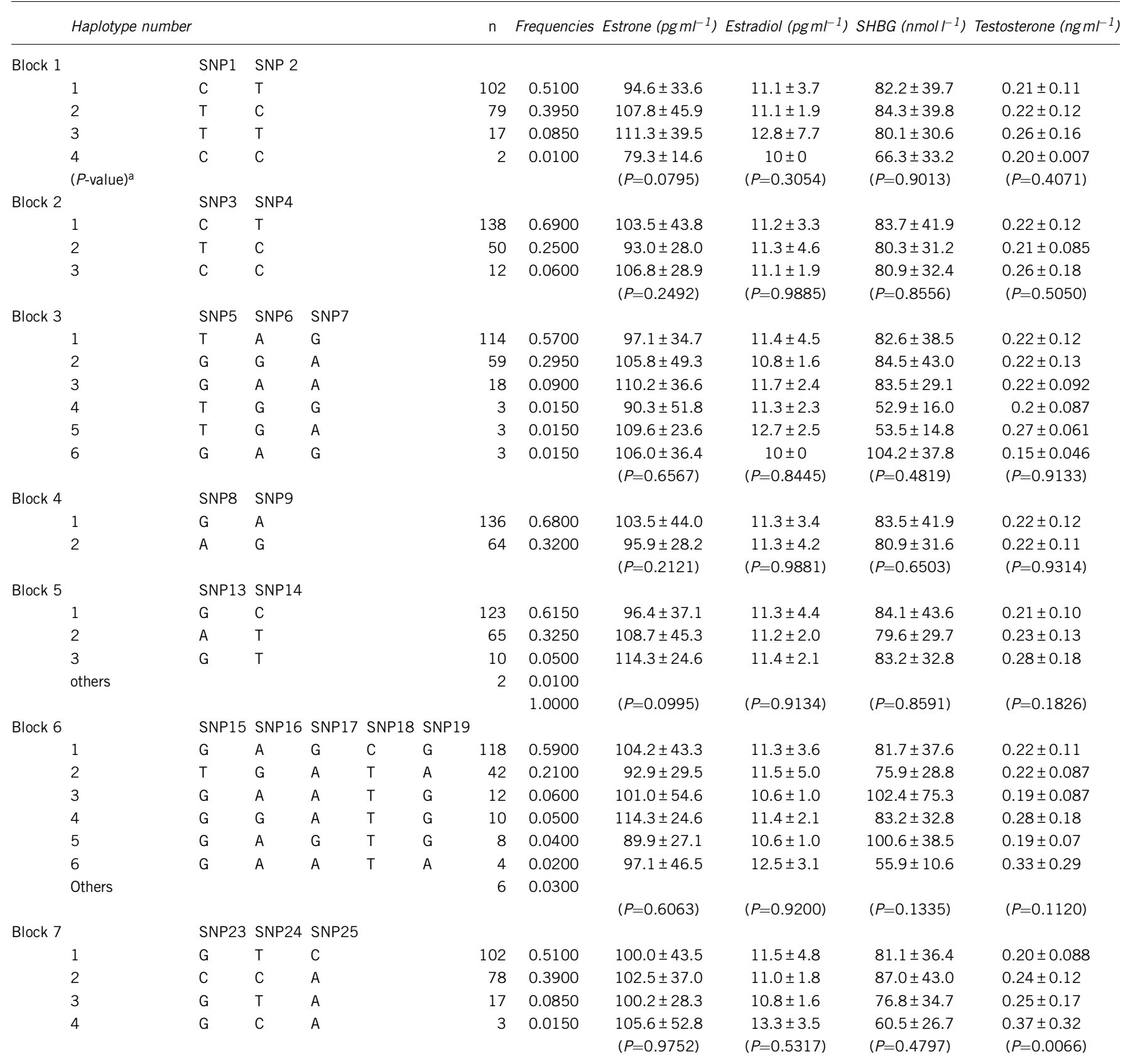

aP-values were tested for equality by the ANOVA test.

But, there are some limitations to this study. In this study, we aimed to identify CYP19A1 loci that showing an association between hormone levels. Significant findings between hormone levels and marker loci do not warrant functional importance of the loci. Therefore, a lack of functional validation is one limitation of the study. The moderate number of cases in this study could be another limitation. Accumulation of evidence from the Asian population is strongly needed.

In conclusion, to the best of our knowledge, this is the first study to examine the association between tag SNPs in CYP19A1 and sex hormone levels in postmenopausal Japanese women. Results showed an association between estrone and testosterone levels and some CYP19A1 loci. These associations might be potential susceptibility markers of hormone-dependent diseases in postmenopausal women. A further study of these associations to determine potential clinical applications is warranted.

\section{ACKNOWLEDGEMENTS}

We are grateful to the assistant staff at the Division of Epidemiology and Prevention at Aichi Cancer Center Research Institute for their support. This study was supported by a grant-in-aid for Scientific Research on Cancer Epidemiology in a Special Priority Area (C) (17015052) from the Ministry of Education, Science, Sports, Culture and Technology of Japan; a grant-in-aid for Scientific Research (C) from the Ministry of Education, Science, Sports, Culture and Technology of Japan; a grant-in-aid for the Third Term Comprehensive 
10-Year Strategy for Cancer Control from the Ministry of Health, Labour and Welfare of Japan; and by a grant-in-aid for Cancer Research from the Ministry of Health, Labour and Welfare of Japan (19-19).

1 Kaaks, R., Rinaldi, S., Key, T. J., Berrino, F., Peeters, P. H., Biessy, C. et al. Postmenopausal serum androgens, oestrogens and breast cancer risk: the European prospective investigation into cancer and nutrition. Endocr. Relat. Cancer 12, 10711082 (2005).

2 Key, T., Appleby, P., Barnes, I. \& Reeves, G. Endogenous sex hormones and breast cancer in postmenopausal women: reanalysis of nine prospective studies. J. Natl Cancer Inst. 94, 606-616 (2002).

3 Lukanova, A., Lundin, E., Micheli, A., Arslan, A., Ferrari, P., Rinaldi, S. et al. Circulating levels of sex steroid hormones and risk of endometrial cancer in postmenopausal women. Int. J. Cancer 108, 425-432 (2004).

4 Labrie, F., Belanger, A., Cusan, L., Gomez, J. L. \& Candas, B. Marked decline in serum concentrations of adrenal $\mathrm{C} 19$ sex steroid precursors and conjugated androgen metabolites during aging. J. Clin. Endocrinol. Metab. 82, 2396-2402 (1997).

5 Simpson, E. R. \& Davis, S. R. Minireview: aromatase and the regulation of estrogen biosynthesis-some new perspectives. Endocrinology 142, 4589-4594 (2001).

6 Misso, M. L., Jang, C., Adams, J., Tran, J., Murata, Y., Bell, R. et al. Adipose aromatase gene expression is greater in older women and is unaffected by postmenopausal estrogen therapy. Menopause 12, 210-215 (2005).

7 Baxter, S. W., Choong, D. Y., Eccles, D. M. \& Campbell, I. G. Polymorphic variation in CYP19 and the risk of breast cancer. Carcinogenesis 22, 347-349 (2001).

8 Berstein, L. M., Imyanitov, E. N., Suspitsin, E. N., Grigoriev, M. Y., Sokolov, E. P., Togo, A. et al. CYP19 gene polymorphism in endometrial cancer patients. J. Cancer Res. Clin. Oncol. 127, 135-138 (2001).

9 Haiman, C. A., Hankinson, S. E., Spiegelman, D., De Vivo, I., Colditz, G. A., Willett, W. C. et al. A tetranucleotide repeat polymorphism in CYP19 and breast cancer risk. Int. J. Cancer 87, 204-210 (2000).

10 Haiman, C. A., Dossus, L., Setiawan, V. W., Stram, D. O., Dunning, A. M. \& Thomas, G. et al. Genetic variation at the CYP19A1 locus predicts circulating estrogen levels but not breast cancer risk in postmenopausal women. Cancer Res. 67, 1893-1897 (2007).

11 Dunning, A. M., Dowsett, M., Healey, C. S., Tee, L., Luben, R. N., Folkerd, E. et al. Polymorphisms associated with circulating sex hormone levels in postmenopausal women. J. Natl Cancer Inst. 96, 936-945 (2004).

12 Parkin, D. M., Whelan, S. L., Ferlay, J., Teppo, L. \& Thomas, D. B. Cancer Incidence in Five Continents, Lyon (2003).

13 Hamajima, N. The Japan Multi-Institutional Collaborative Cohort Study (J-MICC Study) to detect gene-environment interactions for cancer. Asian Pac. J. Cancer Prev. 8, 317-323 (2007).

14 Tajima, K., Hirose, K., Inoue, M., Takezaki, T., Hamajima, N. \& Kuroishi, T. A model of practical cancer prevention for outpatients visiting a hospital: the hospital-based epidemiologic research program at Aichi Cancer Center (HERPACC). Asian Pac. J. Cancer Prev. 1, 35-47 (2000).

15 De La Vega, F. M., Isaac, H. I. \& Scafe, C. R. A tool for selecting SNPs for association studies based on observed linkage disequilibrium patterns. Pac. Symp. Biocomput. 11, 487-498 (2006).

16 The International HapMap Consortium. The International HapMap Project. Nature 426, 789-796 (2003).

17 Thorisson, G. A., Smith, A. V., Krishnan, L. \& Stein, L. D. The international HapMap project web site. Genome Res. 15, 1592-1593 (2005).
18 Livak, K. J. Allelic discrimination using fluorogenic probes and the $5^{\prime}$ nuclease assay. Genet. Anal. 14, 143-149 (1999).

19 Gabriel, S. B., Schaffner, S. F., Nguyen, H., Moore, J. M., Roy, J. \& Blumenstiel, B. et al. The structure of haplotype blocks in the human genome. Science 296, 2225-2229 (2002).

20 Matsuo, K., Wakai, K., Hirose, K., Ito, H., Saito, T. \& Tajima, K. Alcohol dehydrogenase 2 His47Arg polymorphism influences drinking habit independently of aldehyde dehydrogenase 2 Glu487Lys polymorphism: analysis of 2,299 Japanese subjects. Cancer Epidemiol. Biomarkers Prev. 15, 1009-1013 (2006).

21 Suzuki, T., Matsuo, K., Hasegawa, Y., Hiraki, A., Kawase, T., Tanaka, H. et al. Anthropometric factors at age 20 years and risk of thyroid cancer. Cancer Causes Control 19, 1233-1242 (2008).

22 Paynter, R. A., Hankinson, S. E., Colditz, G. A., Kraft, P., Hunter, D. J. \& De Vivo, I. CYP19 (aromatase) haplotypes and endometrial cancer risk. Int. J. Cancer 116, 267-274 (2005).

23 Cai, Q., Kataoka, N., Li, C., Wen, W., Smith, J. R., Gao, Y. T. et al. Haplotype analyses of CYP19A1 gene variants and breast cancer risk: results from the Shanghai Breast Cancer Study. Cancer Epidemiol. Biomarkers Prev. 17, 27-32 (2008).

24 Petry, C. J., Ong, K. K., Michelmore, K. F., Artigas, S., Wingate, D. L., Balen, A. H. et al. Association of aromatase (CYP 19) gene variation with features of hyperandrogenism in two populations of young women. Hum. Reprod. 20, 1837-1843 (2005).

25 Baghaei, F., Rosmond, R., Westberg, L., Hellstrand, M., Eriksson, E., Holm, G. et al. The CYP19 gene and associations with androgens and abdominal obesity in premenopausal women. Obes. Res. 11, 578-585 (2003).

26 Grodin, J. M., Siiteri, P. K. \& MacDonald, P. C. Source of estrogen production in postmenopausal women. J. Clin. Endocrinol. Metab. 36, 207-214 (1973).

27 Bulun, S. E. \& Simpson, E. R. Competitive reverse transcription-polymerase chain reaction analysis indicates that levels of aromatase cytochrome P450 transcripts in adipose tissue of buttocks, thighs, and abdomen of women increase with advancing age. J. Clin. Endocrinol. Metab. 78, 428-432 (1994).

28 McDonald, W. J., Golper, T. A., Mass, R. D., Kendall, J. W., Porter, G. A., Girard, D. E. et al. Adrenocorticotropin-cortisol axis abnormalities in hemodialysis patients. J. Clin. Endocrinol. Metab. 48, 92-95 (1979).

29 Kaye, S. A., Folsom, A. R., Soler, J. T., Prineas, R. J. \& Potter, J. D. Associations of body mass and fat distribution with sex hormone concentrations in postmenopausal women. Int. J. Epidemiol. 20, 151-156 (1991).

30 Kaaks, R., Lukanova, A. \& Kurzer, M. S. Obesity, endogenous hormones, and endometrial cancer risk: a synthetic review. Cancer Epidemiol. Biomarkers Prev. 11, 1531-1543 (2002)

31 Key, T. J., Appleby, P. N., Reeves, G. K., Roddam, A., Dorgan, J. F., Longcope, C. et al. Body mass index, serum sex hormones, and breast cancer risk in postmenopausal women. J. Natl Cancer Inst. 95, 1218-1226 (2003).

32 Judd, H. L., Lucas, W. E. \& Yen, S. S. Serum 17 beta-estradiol and estrone levels in postmenopausal women with and without endometrial cancer. J. Clin. Endocrinol. Metab. 43, 272-278 (1976).

33 Zeleniuch-Jacquotte, A., Akhmedkhanov, A., Kato, I., Koenig, K. L., Shore, R. E., Kim, M. Y. et al. Postmenopausal endogenous oestrogens and risk of endometrial cancer: results of a prospective study. Br. J. Cancer 84, 975-981 (2001).

34 Hankinson, S. E., Willett, W. C., Manson, J. E., Colditz, G. A., Hunter, D. J., Spiegelman, D. et al. Plasma sex steroid hormone levels and risk of breast cancer in postmenopausal women. J. Natl Cancer Inst. 90, 1292-1299 (1998).

35 Thomas, H. V., Key, T. J., Allen, D. S., Moore, J. W., Dowsett, M., Fentiman, I. S. et al. A prospective study of endogenous serum hormone concentrations and breast cancer risk in post-menopausal women on the island of Guernsey. Br. J. Cancer 76, 401-405 (1997). 